\title{
Neurofibromatosis type I and anti-phospholipid antibody syndrome. Report of one case
}

\author{
JOSEF FINSTERER, MD, PHD ${ }^{1}$, CLAUDIA STÖLLBERGER, MD², \\ LILIAN SCHÄFFL-DOWEIK, MD²
}

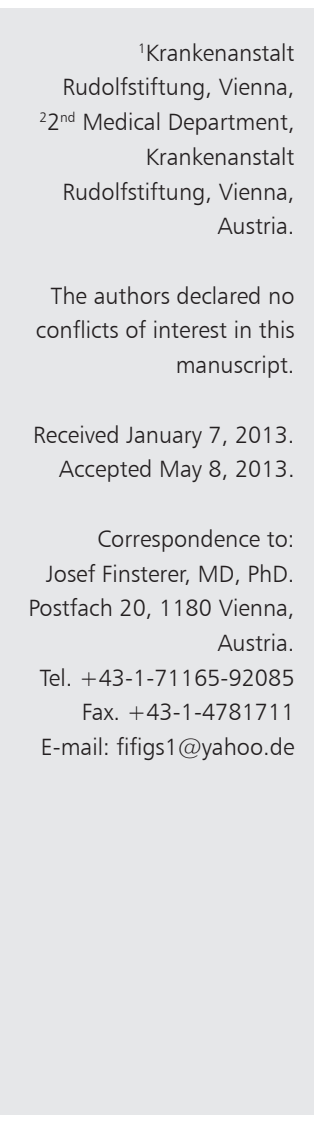

\begin{abstract}
Neurofibromatosis type I (NF1) has been only rarely reported in association with anti-phospholipid syndrome (APS). We report a 38 year-old female with NF1, who developed a cervix carcinoma at the age of 30 years and was successfully treated with conization, without requiring chemotherapy or radiation. She experienced two miscarriages prior to the diagnosis of the carcinoma. When she was 38 years old, an APS was diagnosed based on repeatedly positive lupus anticoagulant tests. The patient continued to smoke and using oral contraceptives. At 38 years of age she had a myocardial infarction, despite the use of oral anticoagulation. She required coronary stenting. Aspirin and clopidrogel were indicated thereafter.
\end{abstract}

(Rev Med Chile 2013; 141: 1068-1071).

Key words: Lupus coagulation inhibitor; Neurofibromatoses; Paraneoplastic syndromes; Thrombophilia.

\section{Neurofibromatosis tipo I y síndrome antifosfolípidos. Informe de un caso}

Es inusual la asociación entre neurofibromatosis tipo I (NF1) y sindrome antifosfolípidos (APS). Presentamos una paciente mujer de 38 años con un NF1 que desarrolló un cáncer cervicouterino a los 30 años y que fue tratada exitosamente con una conización, sin requerir quimioterapia o radiación. La paciente tuvo dos abortos espontáneos antes del diagnóstico del carcinoma. A los 38 años, se le diagnosticó un APS, basado en pruebas de anticoagulante lúpico que resultaron positivas en repetidas oportunidades. La paciente continuó fumando y usando contraceptivos orales y, a pesar de estar con anticoagulantes orales, tuvo un infarto agudo de miocardio a los 38 años. Se colocó un stent coronario y se indicó aspirina y clopidogrel.
$\mathrm{N}$ eurofibromatosis 1 (NF1), also known as "von Recklinghausen disease", is a rare autosomal dominant neuro-cutaneous disorder due to mutations in the neurofibromin gene on chromosome 17q11.2. NF1 occurs with a prevalence of 1 in 4000-5000 individuals and manifests in the skin, bones, arteries, peripheral nerves and central nervous system $(\mathrm{CNS})^{1-3}$. It has been repeatedly reported in association with other disorders, such as multiple sclerosis ${ }^{4}$, or malignancies ${ }^{5}$ but NF1 in association with antiphospholipid syndrome (APS), as in the following case, has been described only twice ${ }^{6}$.

\section{Case report}

The patient is a 38-year-old Caucasian woman, non-smoking, HIV-negative, height 173 
$\mathrm{cm}$, weight $100 \mathrm{~kg}$, with a history of surgery for tendinitis on the left lower arm in 2001, resection of cutaneous fibromas on the radial side of the left lower arm in 2002, and development of caféaux-lait (CAL) macules on the left lower arm since 2003. Since 2006 she developed double vision and flickering and blurred vision of the left eye with which she was able to differentiate only black and white colors since 2009. In 2007 she underwent resection of a fibroma from the right knee. Since 2007 she developed gait disturbance due to a femoral nerve lesion resulting in proximal weakness of the right lower limb. She had a history of migraine and tension headache, both responding to mefenamin acid. She had undergone conisation for a cervical carcinoma in 2003 without requiring chemotherapy or radiotherapy. She had a history of recurrent venous sympathectomy on the left lower arm, for an osteoma within the left frontal sinus, allergic asthma, and for recurrent palpitations since 2008. She had two children and a history of two miscarriages. Both abortions occurred before the carcinoma in 1990 after the gestation had lasted 12 and 16 weeks respectively. During her third pregnancy she experienced a severe septic infection. Since 3 years ago hepatopathy and since 6 months, splenomegaly and thrombocytopenia were noted. In May 2011 APS was diagnosed upon the history and recurrent positivity of the lupus anticoagulant confirmation test (LAC) and the DRVVT test. The lupus-sensitive aPTT was positive once. Antibodies against $\beta_{2}$ glycoprotein and against cardiolipin were not determined. Due to the delayed diagnosis, she received oral anticoagulation only since September 2011. Additionally, she was taking a contraceptive pill since 2003. Her family history was positive for CAL (mother, daughter), recurrent abortions (3 times, mother), pancreas carcinoma (mother), Leyden mutation and deep venous thrombosis (one sister), cardiopulmonary resuscitation at age 6 weeks (son), hepatopathy (2. sister) positivity for the lupus anticoagulans (daugther), immunodeficiency (daugther), ADHS (son), mental retardation (son), hepatoesplenomegaly (son) and coronary heart disease (mother and grandmother).

At age 38 the patient was admitted for anginal chest pain and dyspnea. Diagnostic work-up revealed a myocardial infarction upon the clinical presentation, elevated troponin, and the ECG. Coronary angiography revealed a RCX stenosis and a LAD stenosis, which were treated with two bare metal stents into the RCX and one bare metal stent into the LAD. Secondary antithrombotic prophylaxis included acetyl-salicylic acid and clopidogrel in addition to oral anticoagulation for the APS At age 38 y (November 2011) she experienced a re-stenosis of the right coronary artery requiring ballon dilatation and a drugeluting stent. In the same month a stenosis of the LAD required dilatation and placement of a drug-eluting stent. Because of discontinuations of oral anticoagulation because of panaritiun sugery in May 2012 she experienced an acute occlusion of the LAS requiring a second drug-elutin stent. Blood tests revealed hyperlipidemia, elevated liver function parameters, and thrombocytopenia. The patient did not carry the Leyden mutation. Screening for neurofibromin gene mutations is still under way. Clinical neurologic examination revealed severe visual impairment of the left eye, double vision when looking downwards to the left, sore supra- and infra-orbital nerves in the left side, an exaggerated masseter reflex, bilateral weakness for finger straddling (M5-), brisk tendon reflexes on the upper limbs, lamellar hypoesthesia on the radial side of the left lower arm, CAL spots on the left lower arm, bilateral weakness of the lower limbs with right-sided predominance, hypoesthesia of the lateral right lower limb, and bilateral brisk tendon reflexes. MRI of the cerebrum showed various spots of gliosis in the high parietal region on the right side.

According to the 2006 Consensus Statement, the patient was classified as APS IIa. According to the Sydney Classification Criteria (Table 1: myocardial infarction, one unexplained death of a normal fetus beyond $10^{\text {th }}$ week of gestation, positive antibodies against phospholipids) the diagnosis of APS was considered as confirmed.

\section{Discussion}

The diagnosis of NF1 relies on the presence of at least two of the following criteria: more than one fibroma on or under the skin or one plexiform neurofibroma (large cluster of fibromas involving multiple nerves), freckling of the groin or axilla, more than five CAL spots, skeletal abnormalities such as sphenoid dysplasia or thinning of long bone cortex, Lisch nodules (hamartomas) of the 
Table 1. Sydney Criteria for Diagnosing ALP (diagnosis of ALP requires the presence of at least one clinical and one laboratory criterium)

\begin{abstract}
Clinical criteria
A documented episode of arterial, venous, or small vessel thrombosis other than superficial venous thrombosis in any tissue or organ by objective validated criteria with no significant evidence of inflammation in the vessel wall, and/or

One or more unexplained deaths of a morphologically normal fetus (documented by ultrasound or direct examination of the fetus) at or beyond the $10^{\text {th }}$ week of gestation and/or three or more unexplained consecutive spontaneous abortions before the 10th week of gestation, with maternal anatomic or hormonal abnormalities and paternal and maternal chromosomal causes excluded or at least one premature birth of a morphologically normal neonate before the $34^{\text {th }}$ week of gestation due to eclampsia or severe pre-eclampsia according to standard definitions, or recognized features of placental insufficiency plus
\end{abstract}

\title{
Laboratory criteria
}

Anti-cardiolipin IgG and/or IgM measured by standardized, non-cofactor dependent ELISA on 2 or more occasions, not less than 12 weeks apart; medium or high titre (i.e., > $40 \mathrm{GPL}$ or MPL, or > the 99th percentile) and/or

Anti- $\beta 2$ glycoprotein I lgG and/or IgM measured by standardized ELISA on 2 or more occasions, not less than 12 weeks apart; medium or high titre ( $>$ the 99th percentile) and/or

Lupus anticoagulant detected on 2 occasions not less than 12 weeks apart according to the guidelines of the International Society of Thrombosis and Hemostasis

iris, optic nerve tumors, macrocephaly without hydrocephalus, epilepsy, or posterior lenticular opacity ${ }^{1,7}$. The diagnosis of NF1 in our patient relied on the presence of CAL spots on the left lower arm, fibromas on the left lower arm and the right knee, suspected fibromas on the right lumbar plexus, and the optic nerve glioma. The other diagnostic criteria for NF1 were not fulfilled. A further argument for NF1 was the positive family history for CAL in her mother and daughter.

APS, also known as Lupus anticoagulans syndrome, is an autoimmune coagulation disorder due to the production of auto-antibodies against phospholipids in membranes, cardiolipin, or $\beta 2$ glycoprotein $1^{8}$. APS is characterized by thrombus formation in arteries and veins, manifesting clinically as deep venous thrombosis, ischemic stroke, miscarriage prior to the $10^{\text {th }}$ week of gestation, pre-eclampsia after the $20^{\text {th }}$ week of gestation, or stillbirth (Table 1$)^{9}$. APS may be primary, without other concomitant disease, or secondary, if in conjunction with other autoimmune disorders ${ }^{1,10}$. The diagnosis in our patient was based on the history of two spontaneous abortions and the detection of a positive lupus anticoagulant She had no history of ischemic stroke but had developed myocardial infarction at age 38 . Whether APS contributed to the pathogenesis of the myocardial infarction remains speculative. She was not under oral an- ticoagulation before the infarction but she was smoking, had hyperlipidemia and was taking a contraceptive pill.

Though NF1 has been reported in association with systemic lupus erythematosus $s^{6,11-13}$, and the presence of cardiolipin antibodies ${ }^{6}$, and despite a close link between systemic lupus erythematosus and APS, it has been reported in association with APS only in two cases ${ }^{6,14}$. The first patient had NF1 since childhood but had not yet developed malignancy. He developed multiple ischemic strokes at age 35 being attributed to APS 6 . Though a genetic background also of autoimmune disease is suspected ${ }^{13,14}$, it is questionable if there is a causal relation between NF1 and APS. Arguments for a causal relation are that there are familial cases of APS and that certain polymorphisms are frequently associated with APS ${ }^{15}$. Whether mutated neurofibromin induced the development of the lupus antigoagulant in the sense of a cross reaction remains speculative. Arguments against a causal relation are that APS has been previously reported in association with NF1 only twice ${ }^{6}$, that mutations in the neurofibromin gene were never associated with coagulation defects, and that the clinical presentation is highly variable between the two entities. Possibly, the patient developed the APS in reaction to the carcinoma as a paraneoplastic phenomenon, which has been previously reported 
in a patient with pancreas carcinoma ${ }^{16}$. However, the latency between onset of malignancy and APS was long and onset of the APS was before occurrence of the malignoma. Increased liver function parameters were explained by the reaction of the liver to chronic intake of drugs, coincidental autoimmune hepatitis, or to affection of the liver arteries in the APS. Presumed causes of thrombocytopenia were involvement of the thrombocytes in the APS, splenomegaly, previous sepsis, or side effects of a drug.

This case report shows that primary APS may be associated with NF1. In addition to APS, thromboembolic risk factors such as intake of oral contraceptives, may favor the development of myocardial infarction, which gave the opportunity to discover and investigate the APS.

\section{References}

1. Boyd KP, Korf BR, Theos A. Neurofibromatosis type 1. J Am Acad Dermatol 2009; 61: 1-14.

2. Ferner RE. The neurofibromatoses. Pract Neurol 2010; 10: 82-93.

3. Jett K, Friedman JM. Clinical and genetic aspects of neurofibromatosis 1. Genet Med 2010; 12: 1-11.

4. Pipatpajong $\mathrm{H}$, Phanthumchinda K. Neurofibromatosis type I associated multiple sclerosis. J Med Assoc Thai 2011; 94: 505-10.

5. Dang JD, Cohen PR. Segmental neurofibromatosis and malignancy. Skinmed 2010; 8: 156-9.

6. Carr ME Jr, Howe CW. Lupus anticoagulant and cerebrovascular accident in a patient with neurofibromatosis. South Med J 1989; 82: 921-3.
7. Jouhilahti EM, Peltonen S, Heape AM, Peltonen J. The pathoetiology of neurofibromatosis 1. Am J Pathol 2011; 178: 1932-9.

8. Sangle NA, Smock KJ. Antiphospholipid antibody syndrome. Arch Pathol Lab Med 2011; 135: 1092-6.

9. Koniari I, Siminelakis SN, Baikoussis NG, Papadopoulos G, Goudevenos J, Apostolakis E. Antiphospholipid syndrome; its implication in cardiovascular diseases: a review. J Cardiothorac Surg 2010; 5: 101.

10. Tincani A, Andreoli L, Chighizola C, Meroni PL. The interplay between the antiphospholipid syndrome and systemic lupus erythematosus. Autoimmunity 2009; 42: 257-9.

11. Bitnun S, Bassan H. Letter: Neurofibromatosis and SLE. N Engl J Med 1975; 292: 429-30.

12. Akyüz SG, Caltik A, Bülbül M, Erdo an $O$, Renda R, Demircin G. An unusual pediatric case with neurofibromatosis and systemic lupus erythematosus. Rheumatol Int 2011; (in press)

13. Katzav A, Litvinjuk Y, Pick CG, Blank M, Shoenfeld Y, Sirota P, Chapman J. Genetic and immunological factors interact in a mouse model of CNS antiphospholipid syndrome. Behav Brain Res 2006; 169: 289-93.

14. Xavier de Freitas, CZ, Bisi MC, Sinnott Silva L, Bodanese LC, Staub HL. Neurofibromatosis type I and antiphospholipid syndrome. Rev Med Chile 2010; 138: 1562.

15. Vázquez-Del Mercado M, García-Cobian TA, Muñoz Valle JF, et al. Genotype Ser413/Ser of PAI-2 polymorphism Ser413/Cys is associated with anti-phospholipid syndrome and systemic lupus erythematosus in a familial case: comparison with healthy controls. Scand J Rheumatol 2007; 36: 206-10.

16. Korenkov M, Depta A, Kentner R, Beck J, Junginger T. Paraneoplastic lupus anticoagulans syndrome. Zentralbl Chir 2005; 130: 372-4. 